\title{
Research on Application of ZigBee Technology in Flammable and Explosive Environment
}

\author{
Yang $\mathrm{Li}^{1,2}$, Ke Zhang ${ }^{2}$ \\ ${ }^{1}$ Beijing Jiaotong University, Beijing, China \\ ${ }^{2}$ Beijing institute of Petro-chemical Techmology, Beijing, China \\ E-mail: liyang@bipt.edu.cn \\ Received April 4, 2010; revised May 6, 2010; accepted May 15, 2010
}

\begin{abstract}
Wireless Sensor Network based on ZigBee technology is a wireless network which is composed of many nodes of ZigBee RF chips, sensors and MCUs, especially suitable for application of the remote monitoring system in flammable and explosive environments. This paper presents the characteristics and advantages of ZigBee technology, also discusses the system for hardware and software design. This system effectively fulfills the remote monitoring in flammable and explosive environments and posses high practical values.
\end{abstract}

Keywords: ZigBee, Wireless Sensor Network, Remote Monitoring, Flammable and Explosive

\section{Introduction}

In the oil, chemicals and other inflammable and explosive production environment, it is essential to the production site, some information (such as pressure, temperature, gas concentration, etc.) for data collection and transmission network in order to achieve remote monitoring and control. At present, widely used by cable way through all kinds of information sent to the monitoring center, however, most point of the scene to monitor geographical dispersion, environment, complex terrain, which is encountered in many practical applications: 1) To set up a qualified cable transmission network, there is the complexity of the construction and implementation of the difficulty; 2) In order to achieve all-round production of effective environmental monitoring, dispersed layout must be many types, the large number of nodes to monitor the data acquisition, cable monitoring is often difficult to achieve; 3) The cable monitoring system has its own limitations, such as the laying of a high fixed cost of communication lines. In recent years, the emergence of ZigBee technology has provided some very good ideas to solve these problems mentioned above.

In this paper, the ZigBee technology wireless sensor network system for remote monitoring the production environment of the explosive have been put forward and designed. The system layout of the scene in the production of the sensor node to all kinds of information can be wirelessly sent to the central node, the center node of data through GPRS or RS232 interface module to the monitoring host (PC machine) for remote monitoring of the production environment. The monitoring system has the advantages of low cost, low power consumption, wireless transmission, and reliable communications.

\subsection{ZigBee Technology}

ZigBee is a relatively recent emergence of wireless network communication technology, more than 100 wellknown by the global coalition of hardware and software companies committed to the development of a shortrange, low rate, low-power wireless network standards, the main development direction for the wireless sensor networks, home automation, remote control, industrial automation, agricultural automation, and medical care and other applications.

\subsubsection{ZigBee Technology Features}

Construction of ZigBee-based wireless network technology has the following features:

1) Data transfer rate low: Only $20 \mathrm{k}$ bytes/s to $250 \mathrm{k}$ bytes/sec, to focus on low-delivery applications;

2) Low power consumption: Due to the use of DSSS technology ZigBee replace FHSS technology, and use of hibernation wake-up mechanism for the work of machines, two on the 5th ordinary dry-cell batteries can be used for 6 months to 2 years, which eliminates the frequent replacement of the battery charge or trouble;

3) Low cost: because of low data rate ZigBee, the agreement is simple and royalty-free, so greatly reduced cost;

4) Network capacity: ZigBee Network Node Manager 
may be a number of sub-node, a node can manage up to 254 sub-node. At the same time, the node can be from one network node management, can be composed of 65,536 large-scale network nodes;

5) The short time delay: delay-sensitive applications for optimized, communication delay and activated from hibernation is very short delay, typically $15 \mathrm{~ms}$ latency to 30 milliseconds;

6) Safety: ZigBee provides the data integrity checks and authentication functions, the use of a common encryption algorithm AES2128, while the flexibility to determine their security attributes;

7) Reliable: the mechanism used to avoid collisions, as well as the need for a fixed bandwidth communications business set aside a dedicated time slot, when sending data to avoid competition and conflict; node module automatically between the functions of dynamic network information in the entire ZigBee networks the way through the automatic route for transmission, thus ensuring the reliability of information transmission;

8) Flexible working band: the use of the Channel $2.4 \mathrm{GHz}, 868 \mathrm{MHz}$ (Europe) and $915 \mathrm{MHz}$ (USA), are license-free band.

\subsubsection{ZigBee Architecture}

ZigBee protocol stack structure as shown in Figure 1, IEEE802.15.4 responsible for the physical layer and MAC layer protocol, ZigBee Alliance to develop the network layer, application layer security services interface and protocol, can be user-defined application layer.

1) Physical Layer: the bottom is the agreement to assume a direct role in working with the task of the outside world, is responsible for the modulation data, send and receive work. IEEE 802.15.4 in the physical layer (PHY) defined two criteria, namely $2.4 \mathrm{GHz}$ and $868 / 915 \mathrm{MHz}$ PHY physical layer. The band can be used in the respective channel 16,10 or 1 , each provision of $250 \mathrm{~kb} / \mathrm{s}$, $40 \mathrm{~kb} / \mathrm{s}$ and $20 \mathrm{~kb} / \mathrm{s}$ transfer rate, the physical layer are used in various band direct sequence spread spectrum technology.

2) MAC Layer: The IEEE 802.15.4 protocol definition, the use of the CSMA-CA mechanism to avoid the collision, and its features include wireless links between devices to establish, maintain and disconnect, to confirm the frame mode to send and receive, channel access and control, and rapid automatic Frame Check request reissued, reserved slot management and information management, such as broadcasting. The definition of a radio frame layer, data frames, frames and MAC command confirmation frame, such as four kinds of frame types.

3) Network/Security Layer: The main mechanism responsible for the establishment of networks and management, self-configuration and self-repair function, the realization of the node to join or leave the network, receive or discard the other nodes, as well as to locate and

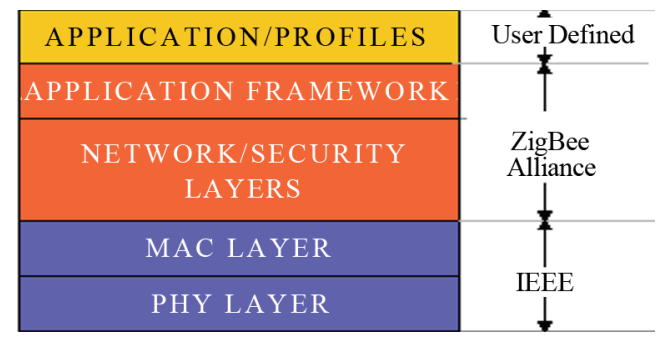

Figure 1. IEEE 802.15.4 stack.

transmit data routing functions, supports a wide range of routing algorithm and a variety of network topology.

4) Application Interface Layer: is responsible for the different applications mapped to the ZigBee network, including security attributes to set up and a number of business data flow and other functions together.

5) Application Layer: The principal objective is the realization of the network communication between different devices, applications and settings for access to information services, calls for the application layer protocol to provide continuous and discrete control applications and other support.

\subsubsection{ZigBee Wireless Network Type}

ZigBee network topology has three: star, cluster tree and mesh type as shown in Figure 2. Among them, the starshaped network is a network-based control center, by a coordinator node and a number of terminal nodes, terminal nodes realized through co-ordination among the communication; cluster tree network has increased the concept of routing, terminal node coordinator can not only access node can access any node has a routing function, it has routing node can not be direct communication between each other only through the co-ordination of the routing nodes between the completion of the communication; mesh network to provide a more flexible mechanism, through the self-organizing routing and wireless data communications to provide multiple paths, when the optimal communication path failure, a redundant mesh network in the other path to choose the most appropriate the path for data communication, therefore, effectively reduce the net-work structure of information transmission delay and improve the reliability of the network communications.

Know from the above, ZigBee network node from the logic function can be divided into co-ordination, routers and terminal equipment, and physical properties from the ZigBee network nodes can be divided into full-featured and simple equipment FFD function device RFD. Among them, the full-function device can act as a coordinator, router or terminal equipment, and can only serve as a simple function of terminal equipment devices.

Each ZigBee network must include a co-ordinator, ZigBee is the network coordinator of the center responsible for the organization and maintenance of the network by 


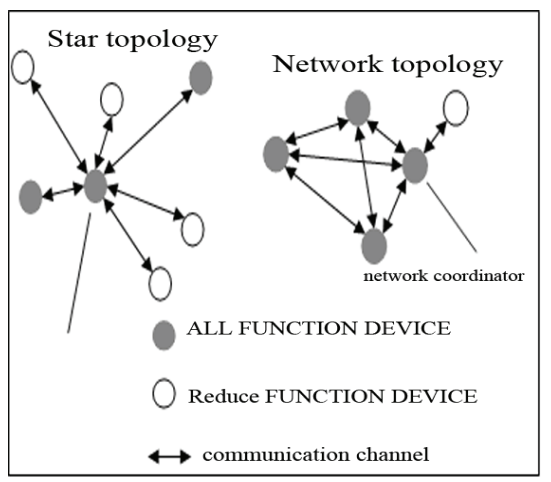

Figure 2. ZigBee topology.

adding a new node and the allocation of 16-bit short address; Zigbee routers responsible for routing node, and used to expand the scope of the network; and the terminal equipment is to achieve a specific functional unit.

\section{Explosive Production Environment Remote Monitoring System Architecture}

System structure shown in Figure 3, the entire system by monitoring the host, GPRS module (or, a ZigBee coordinator node, a number of ZigBee routers, ZigBee nodes and a number of nodes of terminal equipment. This is a cluster tree network structure is conducive to the number of network nodes and the physical expansion of the scope, complex, multi-node wireless network communication system is also an important reference value.

The co-ordination of the network nodes, network management functions, the receiving terminal device node for the data upload, and transfer through the GPRS network to the monitoring center. Router nodes for routing of information transmitted, allowing other nodes join the network. Node device to the network coordinator from time to time collect information to send and receive commands from the monitoring host. ZigBee module used for GPRS networks and Internet networks, the Internet (also available in other ways), the realization of ZigBee network datas to monitor the upload and download the host commands. Host real-time monitoring of the collection, storage, monitoring and processing equipment from a remote terminal nodes of information, and can overrun the police at any time, such as setting parameters for the production environment to achieve effective monitoring and management, its functions are divided into two major parts, 1) Data Monitoring: to receive from the ZigBee network information collected, the corresponding data into the database; to receive instructions from the managers, and command frame format in accordance with the configuration commands, GPRS module through the command issued to the ZigBee network and do the action. 2) Data Management: The database can be found, query data from the current

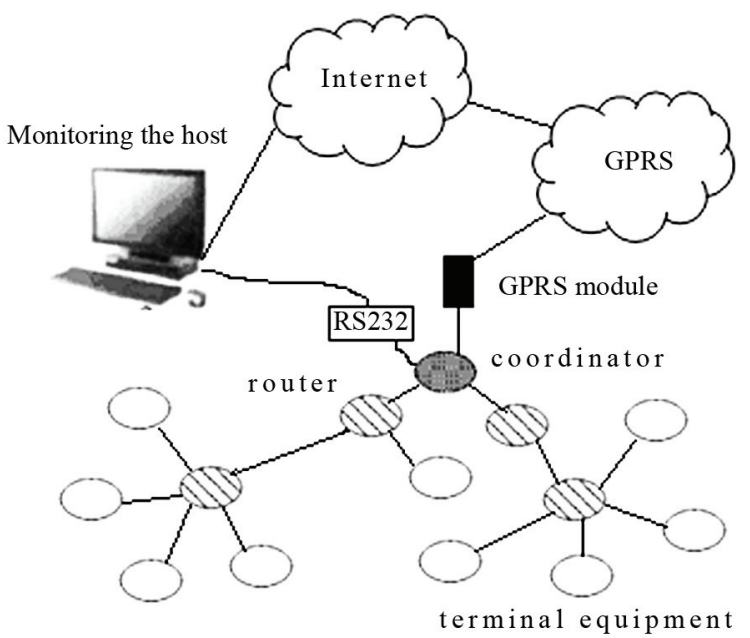

Figure 3. Structure of the framework of remote monitoring system.

ZigBee network information, such as: the production of the ambient temperature, pressure, overrun alarm, such as the peak period.

ZigBee end-node using the occasional wake-up call from time to time work, time to wake up from hibernation to start data acquisition, ZigBee routing node to send a message, send completed and then enter hibernation. ZigBee routing nodes will collect the data sent to the ZigBee coordinator node, gateway GPRS module through the data uploaded to the remote monitoring center.

\section{System Hardware Design}

Explosive production environment is a remote monitoring system by a number of ZigBee network node. Each node is basically the same hardware structure, but not the same network layer. ZigBee hardware in each node has two basic components: micro-controller and wireless receiver send some. Hardware-specific features into the single chip to achieve by the burning process to decide.

To facilitate the design and cost savings, the system uses a wireless transceiver and controller integrated with the CC2430 single-chip solution module, the module from a Norwegian company Chipcon is in line with standard IEEE 802.15.4-chip ZigBee products. It incorporates a single chip ZigBee radio frequency (RF) frontend, memory and microcontroller. It uses an 8-bit MCU (8051), and $128 \mathrm{~kb}$ with a programmable flash memory and $8 \mathrm{~kb}$ of RAM, also includes analog-to-digital converter (ADC), 4 timers (Timer), watchdog timer, $32 \mathrm{kHz}$ crystal oscillator of the sleep mode timer, power-on reset circuit, power-fail detection circuit, as well as 21 programmable $\mathrm{I} / \mathrm{O}$ pin. Its block diagram is shown in Figure 4. 


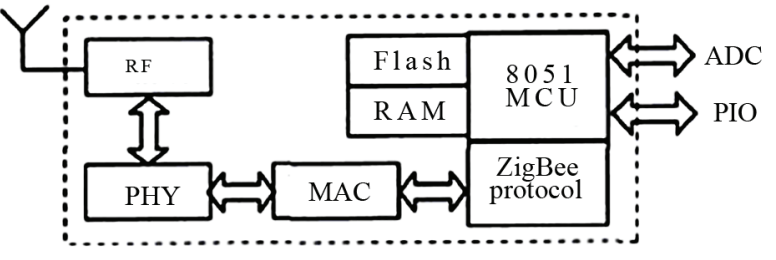

Figure 4. CC2430 structure.

CC2430 network, send and receive data is built on the ZigBee protocol stack based on. If we are to use the CC2430 ZigBee chip, first of all be familiar with the ZigBee protocol stack (see Figure 1), based on the actual needs of the application layer programming. At present, TI has fully disclosed the ZigBee protocol stack CC2430 proceedings; it is relatively easy program development process to achieve. FLASH memory in the CC2430 in the internal processor to run the application, when the system is activated, the chip will be stored in FLASH in the program in the implementation of the RAM.

CC2430 chip with only a small number of external components to send and receive signals will be able to achieve functional, very simple and practical. ZigBee nodes of the system hardware structure diagram as shown.

According to actual needs of each node can choose a number of different sensors (such as temperature, pressure, etc.) to meet the requirements of the monitoring points. Coordinator node GPRS module also need to host and monitor connected to RS232 interface can also be used to connect the host and monitor (if communication from the close), chip-level conversion MX3232. Node power circuit terminals using two alkaline batteries on the 5 th, as the coordinator node transceiver has been in a state, so the use of external power supply.

\section{System Software Design}

ZigBee protocol stack to provide a number of Application Programming Interface, such as aplFormNetwork (), aplJoinNetwork (), aplSendMSG() function and so

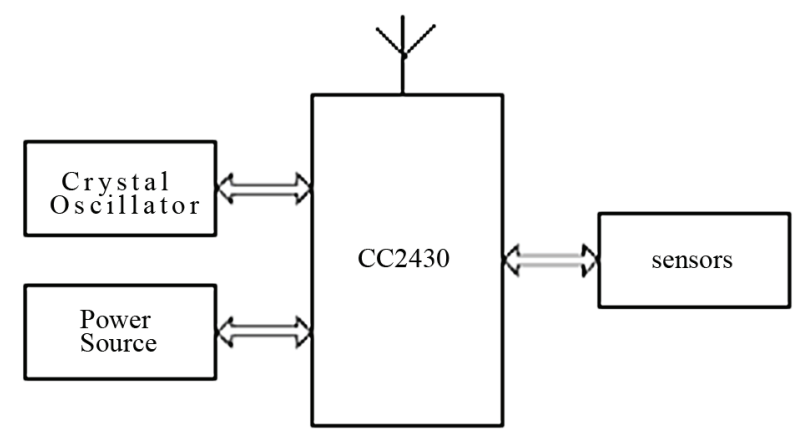

Figure 5. ZigBee node hardware structure. on, the user can call these functions to prepare their own applications.

System Software primarily to achieve two basic functions: 1) to achieve a co-ordinator node and other network nodes and communications; 2) cycle to achieve the various nodes and send sensor data acquisition.

\subsection{Node Design Coordinator}

Network as the network coordinator of the center is responsible for the establishment of a network, information reception, aggregation, processing and sending control instructions and implementation. Coordinator power to start the procedure after the initialization, by calling the function aplFormNetwork ()create a network, select a Coordinator PANID as a network logo, create a routing table, and then released to inform the other routers broadcast frame or a terminal node The addition of node equipment. Start by sending a GPRS module AT command set serial communication rate, the establishment of data communication socket connections ready to begin to send and receive data and instructions to implement various operations. Process flow chart is shown in Figure 6.

\subsection{Terminal Node Programming}

Terminal node network is mainly responsible for a variety of information (such as temperature, pressure, etc.) to collect data to send and receive commands to control the implementation. End-node power-start initialization process

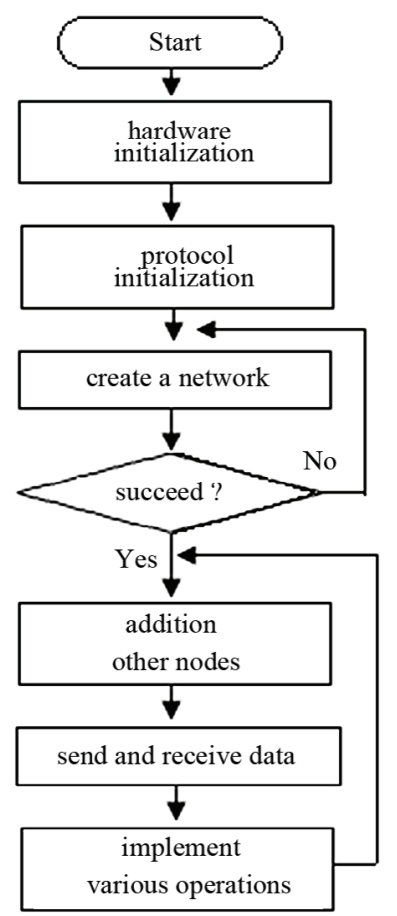

Figure 6. Flow chart of the program coordinator node. 
by calling the function aplJoinNetwork () to join the network, active and effective network of channel scanning, the nearest coordinator to find a suitable node or router node apply to join the network, access is approved, the beginning of information gathering send and receive instruction implementation. Terminal node to take a cyclical mode, do not work in a dormant, down, low-power to achieve energy-saving effect.

Part of the node device code is as follows:

void main (void) \{

hallnit ( ) ; // hardware initialization

apllnit ( ) ; // protocol stack initialization

do \{

aplJoinNetwork ( ) ; // join the network

while (apsBusy ( ) ) $\{\operatorname{apsFSM}() ;\}$

\}

while (aplGetStatus ()$=$ WXLPAN_STATUS SUCCESS ) ;

while (1) $\{\operatorname{apsFSM}() ;\}$

\}

\section{Conclusions}

In this paper, the design of ZigBee technology production environment explosive remote monitoring system, the degree of coverage cluster tree network structure, node chip CC2430, has flexible, economic practicability, design easy. If the target needs to be identified in accordance with the actual sensor types and routers and the number of terminal nodes, the system can be applied to the production and living more occasions to address the practical application of the cost of wired network cabling is too high, not the arrival of the regional environment kinds of information to monitor the problem.

\section{References}

[1] J. A. Gutierrez, "Wireless Medium Access Control (MAC) and Physical Layer (PHY) Specifications for Low Rate Wireless Personal Area Networks (LR-WPANs), (IEEE Standarted for Information Technology802.15.4.)," Institute of Electrical \& Electronics, 2003.

[2] P. S. Neelakanta and H. Dighe, "Robust Factory Wireless Communicat Ions: A Performance Appraisal of the Bluetooth and the ZigBee Collocated on an Indust Rial Floor," IEEE Computer Society, Vol. 3, 2003, pp. 2381-2386.

[3] Chipcon, "CC2430 Preliminary Data Sheet (Rev. 1.03) SWRS036A,” Chipcon, 2005.

[4] R. Reese, “A ZigBee TM-Subset/IEEE 802.15.4 TM Multiplatform Protocol Stack," Electrical/Computer Engr MSU, 2006.

[5] “ZigBee Protocol Specification," http://www.ZigBee.org 